2019-10-25

\title{
Quality of Experience (QoE) in Cloud Gaming Architectures: A Review
}

\section{Laghari, AA}

http://hdl.handle.net/10026.1/14894

10.3233/MGS-190313

Multiagent and Grid Systems - An International Journal IOS Press

All content in PEARL is protected by copyright law. Author manuscripts are made available in accordance with publisher policies. Please cite only the published version using the details provided on the item record or document. In the absence of an open licence (e.g. Creative Commons), permissions for further reuse of content should be sought from the publisher or author. 


\title{
Quality of Experience (QoE) in Cloud Gaming Architectures: A Review
}

\author{
Asif Ali Laghari, Hui He, Kamran Ali Memon, Rashid Ali Laghari, Imtiaz Ali Halepoto, Asiya Khan \\ Computer Networks and Security Lab, School of Computer Science \& Technology \\ Harbin Institute of Technology \\ School of Mechatronics, Harbin Institute of Technology
}

State Key Laboratory of Information Photonics and Optical Communications, School of Electronic Engineering, Beijing University of Posts and Telecommunications, 100876, China

QUEST Nawabshah, Sindh, Pakistan

School of Engineering, University of Plymouth, Plymouth United Kingdom

asiflaghari,hehui, [@hit.edu.cn], ali.kamran77@gmail.com, rashidali@stu.hit.edu.cn, Halepoto@quest.edu.pk, asiya.khan@plymouth.ac.uk

\begin{abstract}
Cloud gaming is a new way of online gaming, which renders the game data on the cloud side instead of the end user's system and is forwarded via a high-speed network. In cloud gaming; game software and emulators run on the high-speed server of cloud and services available for users on the commercial and free basis. Users can use rendered data of game from the cloud using thin heterogeneous devices via the Internet to play the game anytime and anywhere. The popularity of cloud gaming has increased since the late 2000s which attract the industry and academia. Quality of Experience (QoE) domain has been added in cloud gaming architectures to assess user satisfaction, enjoyment, and needs during online gaming. In this research paper; we survey and analyze the previous cloud gaming models and architectures and offer aspects of future development; which will help to give the quality of service (QoS) according to service level agreement (SLA) and increase user satisfaction level for cloud gaming, hence, improving the overall QoE.
\end{abstract}

Index Terms - Quality of Experience (QoE); Quality of Service (QoS); Network parameters; Cloud gaming

\section{INTRODUCTION}

Nowadays online gaming is growing in popularity on the Internet. According to a recent study and forecast [1], global Internet online gaming traffic was accounted for 126 petabytes per month in 2016 and by 2017; it is expected to reach 189 petabytes per month. The overall gaming includes casual online gaming, networked console gaming, and multiplayer virtual-world gaming. The online gaming started in the 1980s and MUD1 was the first game, which was created in 1978 and limited to an internal network before connected to ARPANET in 1980 [2]. After that commercial gaming started in the next decades and the first commercial online game was "Islands of Kesmai" and more graphical games were launched such as "MSX LINKS" actions games in 1986 [3].

In the past client, server gaming was popular among the users, rendering of graphics and games executed at user's device, the server provided a game program and network facility for sharing the video at both 
ends for multi-player scenario [4]. Client-server gaming did not satisfy the user and they faced problems because of the graphics rendering of the game required high processing and memory units [5]. Users also face device configuration issues in multi-player; one user has high-performance computing device and others have low performance, resulting in waiting and slow gaming. Users required a purchase to different game platforms; configure additional hardware which increased the cost of end-user for playing PC based and online games [6]. Price of a legal copy of the game program, the context of games and user devices for accessing and rendering of data also has an impact on the user's QoE.

The cloud gaming has started in 1997 with the launch of Ultimate "Online" [7]. Cloud gaming technology is different from traditional online gaming because the games are rendered and processed in the cloud server and video is forwarded to end users and player required the thin client to play the games [8]. Users received the video frames from the cloud for view game video and send input data to a cloud server, as shown in the cloud gaming model is shown in figure 1 . Shows the user sends commands to play the game and receives display from a cloud data center.

The user did not need more hardware because all complex rendering of games handled by cloud servers, which decrease the burden from user devices and time of processing [9]. Cloud servers offer platform independent games so the user can play the similar game using any device, anyplace in the world. Cloud servers stop users using a pirated version of games on their own devices. This supports the developer to get data associated to the game exist in cloud servers, which will be used to update the game. The developers can update the games on the cloud and changes are automatically reflected in the users. Cloud servers required the user experience to improve their services for clients who pay for games, so the QoE is used to capture the user feedback about the cloud services [10].

The definition of QoE is given by Qualinet as "Quality of Experience (QoE) is the degree of delight or annoyance of the user of an application or service. It results from the fulfillment of his or her expectations with respect to the utility and/or enjoyment of the application or service in the light of the user's personality and current state" [11]. QoE is a term used to measure a user's level of satisfaction and enjoyment for a particular product or service [12]. QoE can be captured in two techniques, subjective QoE based on the questionnaire, interviews and scales and objective QoE based on the technical Quality of Service (QoS) data and objective human factors which are related to the human physiological and cognitive system [13]. QoE is measured in the Mean Opinion Score (MOS) which is scored by users subjectively.

\section{Cloud Gaming Model}

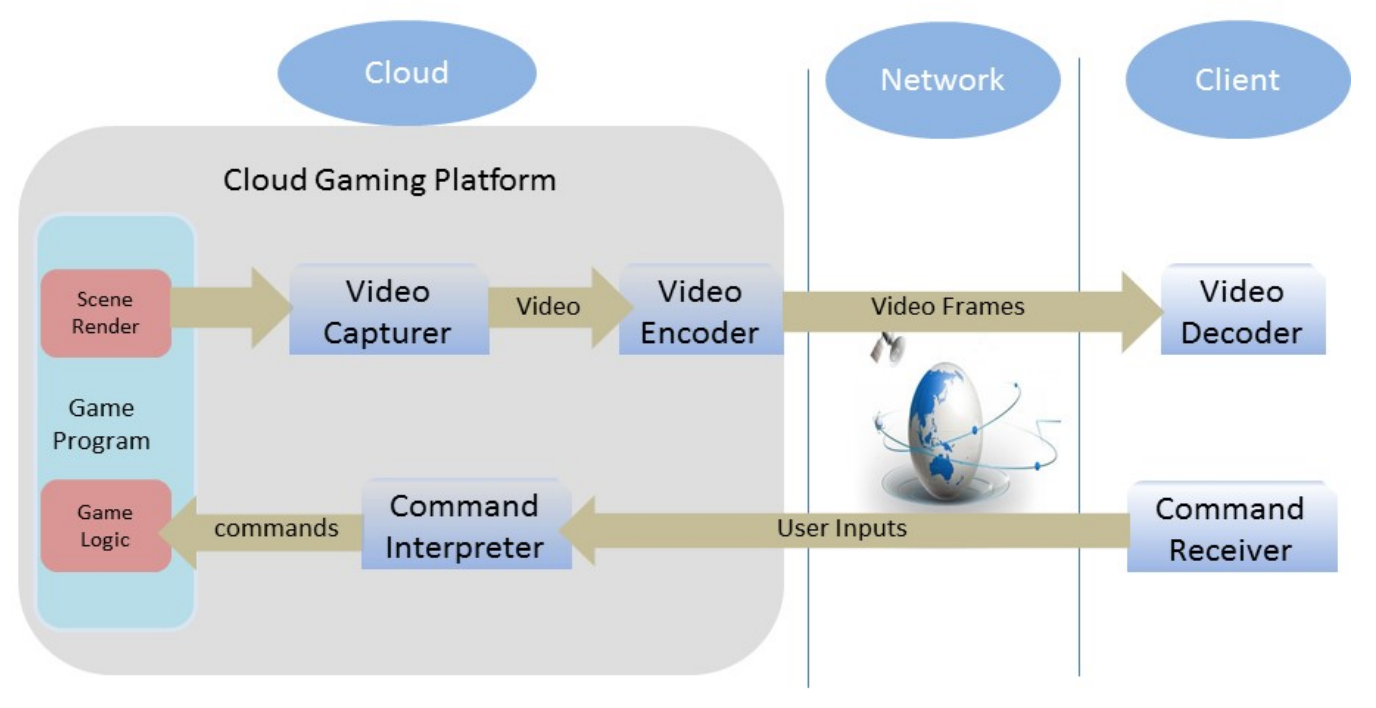


Fig 1. Cloud Gaming Model

- The paper addresses the topic of QoE in cloud gaming architectures, background of QoE and its involvement in cloud gaming to assess the end user's satisfaction and enjoyment level.

- This provides a review of both objective and subjective QoE measuring procedures for cloud gaming and frameworks, such as framework analysis and comparison. Restriction of past work and difficulties of future work additionally examined.

- Further, we distinguish and talk about a lot of open issues yet to be tended to, for precise estimation of QoE in cloud gaming. This work will help us in identifying the main QoS parameters in cloud gaming and developing QoE enabled cloud gaming platform.

The rest of the paper prepared in five sections. In section II we give previous cloud gaming QoE provision architectures, section III is based on the consideration of QoE provision in Cloud gaming. Section IV provides open issues for future research and finally, in section V we conclude the work.

\section{OVERVIEW CLOUD GAMING ARCHITECTURES WITH DIFFERENT APPROACHES}

QoE domain has been merged in cloud gaming architectures to get feedback from users about their satisfaction level and needs. Cloud computing provides three layers, such as software-as-a-service (SaaS), platform-as-a-service (PaaS) and infrastructure-as-a-service (IaaS). Gaming-as-a-service (GaaS) was introduced by developers to offer online gaming as a service to end users. GaaS allow rendering of game data at the cloud side and send results to end users via the high-speed Internet to remote locations [14, 15]. Cloud computing make gaming easy for end users and they can play without having their own highperformance computing system and licensed software, the user only required a network connection, display LCD and controlling the keyboard. Cloud gaming is popular due to the development of gaming platforms such as AppStream [16], StreamMyGame and GamingAny-Where. Slow Internet speed is an issue for playing cloud gaming, cloud supports low frames per second (FPS) gaming [17, 18], which decreases the QoE of the game players. HD gaming requires high bandwidth for sending data from cloud to end user, but unfortunately, the dynamic nature of network traffic and limited network bandwidth is a problem. Several researchers provided a solution to achieve gaming with good QoE of users by considering network bandwidth in architecture development [19, 20,21].

Cloud gaming architectures were developed with different approaches such as local rendering and remote rendering models [22, 23, 38], QoE based models and video quality adjusting models. Remote rendering models provide the facility to play games without high processing hardware. The server collects instructions from users and sends to the cloud for game rendering where actions and rendering performed on the actions of users. Rendered data of a game is sent via the network from cloud to end users; in this scenario, the network bottleneck is a problem to play conventional gaming for end users [23].

Cloud gaming architecture and platforms are developed with different tactics to split the task between clients and cloud servers. Few platforms support streaming graphics from cloud servers to clients and graphics rendered at client's GPU [24, 25]. In these types of platforms, the client requires powerful GPU to process the high-quality game scene in real time, which is not good for limited resources devices such as mobile devices and set-top boxes. Platforms support post rendering operations less part is rendered at 
the client side and the rest of 3D rendered is done at the cloud side [26, 27]. Client rendered includes lights, augmenting and texture as post rendering operations. These approaches to game platforms increased the development cost of the game and complicate the development of the game. In video streaming architectures of games, cloud rendered the game scene and stream video to the client $[20,28]$. Video streaming approach is lighter weight compared to the other approaches of game architectures because in this approach client only decode and display videos which need low resources, support heterogeneous devices, easier to make and requires the smallest augmentations on game code.

Main commercial video streaming cloud gaming platforms are the GaiKai [29], Ubitus [30] and OnLive [31]. GamingAnyWhere is open, cross-platform, efficient and modularized. GamingAnyWhere has influenced many research projects on cloud gaming, such as GPU consolidation [32], e-learning applications [33] and cloud resource allocation [34].

\section{CLOUD GAMING QOE PROVISION ARCHITECTURES}

The delivery of cloud gaming services with QoS according to user's QoE requires the support of architectures, applications, and protocols so that game users can use cloud gaming services ubiquitously: anytime and anywhere [35, 36]. Cloud gaming communication needs to meet the requirements, such as high bandwidth network, low packet delay and loss, low error rate, the acceptable frame rate of video and very small delay variance [37]. QoE based architectures and models for cloud gaming were proposed by researchers by considering different parameter such as video rate, multiplayer platform performance, bandwidth, virtual machine placement, etc. for assessment of end-user QoE.

This section covers the analysis of video streaming based and network based approaches for cloud gaming.

\subsection{Analysis of video streaming based approaches for cloud gaming}

The recent researches compress the video without compromising the quality of video and give QoS according to the network bandwidth and latency. One such work provided by Sun and $\mathrm{Wu}$ [41] and a new bit allocation scheme is proposed on the MB layer based on ROI (Region of Interest) for improving bandwidth efficiency. ROI is a particular area of interest where the user focuses during playing the game, in car racing gaming track, dashboard and ranking is ROI, but the background is not-ROI. The scheme is based on reducing bits of non ROI using a proposed algorithm and forward to game users. In the proposed scheme ROI and importance of value are identical because the user may be interested in object more than ROI and that object may not be in the region of the screen. Important values of the object are detected and assigned to the graphics processor and ROI values of every pixel can be generated along with the original rendered picture. After that rate control (RC) module will use produced ROI map to implement ROIbased rate control. Objective QoE based method is also proposed with rate control scheme by using integrated mean squared error (IMSE) because peak signal to noise ratio (PSNR) is not suitable to measure the performance of ROI-based image. This approach is better to compress trivial areas of the frame and manage the bit rate for slow networks, but compressing scheme will not know ROI of the particular user because every user has different interests in playing the same game. This approach will not improve the overall QoE of game users. 
Cloud gaming experience was analyzed Hong et al. by extending GamingAnywhere (GA) platform in four sections such as servers, clients, portals and a manager [44]. Crowdsourcing system was built on the GA and subjective ratings of users were collected by using online questionnaire on different games such as (Batman, FGPX, CoD) having different frame rates $(10,20,30 \mathrm{fps})$ and bitrate $(0.5,1,2 \mathrm{Mbps})$. Results show that higher bit rate with a fixed frame rate provides higher MOS, the significance of sharing bandwidth among the game users. During the experiments, researchers got several new findings such as high frame rate make games more responsive and provide higher interactivity, but high frame rates video required more frames to encode this cause's lower graphics quality. When bitrate rate is low $(0.5 \mathrm{MB} / \mathrm{s})$ and the frame rate is high then overall MOS was low, but the frame rate is high and bitrate $(2 \mathrm{MB} / \mathrm{s})$ is also high then MOS is high so the high frame rate is not acceptable because it also causes low graphics quality. Two algorithms were proposed to adopt the video rate in dynamic networks. Analytical results show that algorithms run in polynomial time and achieve ideal adaptation. Simulation results fulfill according to analysis and proposed algorithms achieve users required optimization criteria, can be scaled more for 8000 gamers for problem solutions; outperform the baseline algorithms by up to $46 \%$ and $30 \%$. The author provides new findings during the experiments and analytical results of the algorithm were presented with no real-time implementation and simulations results.

End-to-End (E2E) lag in video games models was presented by Metzger et al. [45] for online and cloud variants. The lag model is given for collecting Quality of Experience (QoE), particularly for video games. The lag model notifies time passed between a player input event keystroke or mouse movement and effect on the display event in the local display. E2E Lag depends on the video codec delay for cloud games, the frame rate and server tickrate, processing time, network delay and command rate, which is shown in figure 2 .

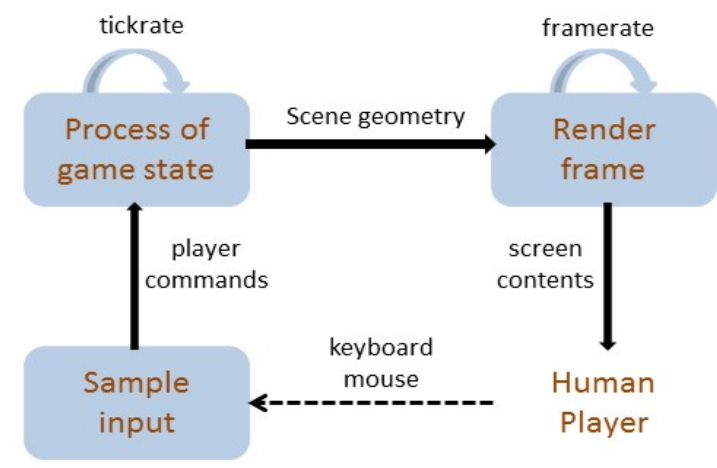

Fig 2. Basic model of a continuous main video game loop [45].

The model is simulated using Discrete Event Simulation (DES), showing the dominant influence of the game frame rate on the E2E lag, particularly for low frame rates. This model explains the lag of different type of games and is important for the design of game QoE assessment.

\subsection{Analysis of Network based approaches for cloud gaming}

An Open Cloud Gaming System, GamingAny-where was given by Huang et al [38]. The proposed gaming system GamingAnywhere is efficient and provides high responsiveness and video quality as compared to the earlier gaming systems such as OnLive and StreamMyGame. The GamingAnywhere system is available for Windows and Linux and OS X, but it does not support Android and iOS. The 
system can support PC based and online games to play via thin clients. This gaming system is extensible so the developer can replace video codecs and network protocols and extend furthermore according to game users. The portable feature provides the facility to the user can use wireless network and client using different operating systems and configuration made easier to use proposed GamingAnyWhere system for gaming or online video streaming with diverse parameters. The text-based configuration files also available for the user, so they can change parameters for best combinations, which fit their system according to usage scenario. This platform is openly available for developer and researcher to develop further and implement in a real-time cloud environment for community and commercial based gaming.

Network speed is a major issue in the delivery of video data of game in GamingAnywhere although it reduces the network delay compare to previous cloud gaming architectures, so avoiding network speed issue new Cloudfog lightweight system is proposed [39]. In this proposed gaming system, supernodes are used as a fog-based concept in the middle between the cloud and end users. Cloud processing will rendering game graphics and overall computing of the virtual world's new state and updating to supernodes and connecting each user to nearby game data supernodes. The user did not have a fast network link to connect with cloud and received a virtual world update and rendered game and cloud videos that did not forward the whole video of game to long-distance users. This method provisions high QoE on slow networks and shortens the reply time, upsurge user coverage and decrease network price. The Cloudfog was compared with GamingAnywhere: [38] and EdgeCloud [40] models and it provide better QoS for a game without a long delay. In CloudFog customers are really close to supernodes, so customer received videos from near supernodes in its place of long distance servers.

The majority of research work has been done on the network bandwidth consumption and video frame rate because game video broadcasted to users at $60 \mathrm{fps}$ and limited network bandwidth does not support smooth video delivery to end users $[39,41,42,43]$. High video quality and low network bandwidth cause delay packet delivery at end users from the cloud, so the wait time, buffering and delay input actions from user degrade the user experience.

The network is also important for playing cloud gaming, which is a bridge between the client and the cloud server for data transfer. Network traffic is dynamic so difficult for the high data rate to receive every time due to peak usage of the network [46].

The influence of network factors like packet delay and loss were analyzed by different researchers. Jarschel et al. [47] measured subjective QoE to examine the impact of the loss and delay of the network taking part in cloud gaming on the consumer experience. Three games had been chosen Pro Evolution Soccer for the omnipresent point of view (slow-pace gameplay), Final Fantasy XIII for the 3rd person point of view (medium-paced gameplay) and Gran Turismo HD Concept for the 1 st person perspective (fast-paced gameplay). The reason of choosing a variety of three video games was to supply participant extra picks to repeat tests and give scores for the video games on the unique network loss and delay from the cloud to client and consumer to cloud server ongoing traffic. Network traffic was disturbed by used NetEm emulator, which was part of test-bed and middle between cloud gaming server and the end user. The results founded on the 79 test runs, 790 users assigned MOS for cloud gaming. The MOS esteems fluctuate from game to game; moderate moving game (video contents) improves client appraisals than the quick moving substance game and medium speed moving substance game get reasonable evaluations. The effect of packet loss and delay on cloud gaming is like its impact on 
traditional gaming. The system delay (bi-directional) was expanded then client MOS esteems were diminished in light of the fact that clients hang tight for a response in the game based on their response. Players of quick diversions acknowledge the high loss when contrasted with high delay in light of the fact that for player's achievement in the game, it is extremely solid to respond rapidly. Amid the review, just $15 \%$ of clients were eager to pay a month to month charge for business cloud gaming if the QoS is given to them.

The other subjective QoE assessment of In-home streaming of online gaming was done by Slivar et al [48] on (World of Warcraft) game by using Gam-inganywhere platform. The research was based on the QoE degradation of the different networks for traditional online gaming and In-home gaming. The results show the packet loss and delay have a major impact on the gaming QoE and packet loss parameter have more influence on the QoE as compared to the packet delay. Transferring from traditional online gaming (client-server) to solely cloud gaming degrades the QoE of the game player due to network QoS. This may result in the inexperienced player to not play the game in the degraded network condition as compared to inexperienced players. User feedback proof that in-home gaming is possible if better video quality is guaranteed during the streaming.

Considering the network bandwidth issue in cloud gaming, a new game architecture was proposed by Muhammad et al. [8]. The model is based on a cloud-end and client-end; it consumes low bandwidth as compared to typical remote rendering cloud models. This model based on the communication of cloud and client end is different from typical cloud model to send rendered frame results to the client, but it will send/receive a number of commands that will allow a game to be played at user side for a short period. The brick breaking game is used to simulate the proposed model with a mixture of Amazon Elastic Computing (EC2) and Relational Database (RDS) instances. Client input is gathered for various situations through subjective examinations and the exhibition is worthy. Game players advised proposed gaming architecture produces only $1 \mathrm{kbs}$ data per user. This model is good for cloud gaming slow speed networks for short time period, but also the drawback of this model is that it does not support continued gaming for a long time.

\section{QUALITATIVE/QUANTITATIVE COMPARISON OF THE FEATURES AND PERFORMANCE OF THE CLOUD GAMING ARCHITECTURES.}

In this section a relative study of the current QoE based cloud gaming frameworks and architectures as far as different qualities, for example, arrangement factors, examination support, revealing and kinds of QoE support as given in Table 1. Deployment parameter is the core parameter of cloud gaming architectures. Mostly developed architectures support quantitative analysis of data although their parameters differ from each other. Cloudlet [41] use the peak signal to noise ratio (PSNR) parameter to assess the QoE and performance of architectures by collecting objective QoE but did not support reporting function the QoE degradation. Adaptive cloud [44] uses subjective QoE assessment of video quality of games on different bitrate and codecs. This architecture also supports the quantitative analysis of data without reporting function. E2E [45] calculate the round trip time (RTT) of packets and subjective QoE assessment of video quality with the quantitative support of data analysis. Jarschel [47], Empirical QoE[48] and friendly cloud gaming used NQoS/AQoS parameters to assess the QoE of games and uses subjective QoE to assessment for performance measurement. 
TABLE I. COMPARISON OF QOE CLOUD GAMING ARCHITECTURES WITH DIFFERENT PARAMETERS AND FEATURES

\begin{tabular}{|c|c|c|c|c|}
\hline $\begin{array}{c}\text { Cloud Gaming QoE } \\
\text { Architectures }\end{array}$ & Parameters & $\begin{array}{l}\text { Analysis } \\
\text { Support }\end{array}$ & Reporting & Remarks \\
\hline Cloudlet [41] & PSNR & Quantitative & No & $\begin{array}{l}\text { Objective } \\
\text { Evaluation }\end{array}$ \\
\hline Adaptive cloud [44] & $\begin{array}{l}\text { Video } \\
\text { codecs }\end{array}$ & Quantitative & No & $\begin{array}{l}\text { Subjective } \\
\text { Evaluation }\end{array}$ \\
\hline E2E [45] & $\begin{array}{c}\text { Video } \\
\text { Quality \& } \\
\text { RTT }\end{array}$ & Quantitative & No & $\begin{array}{l}\text { Subjective } \\
\text { Evaluation }\end{array}$ \\
\hline Jarschel [47] & $\begin{array}{c}\text { NQoS \& } \\
\text { AQoS }\end{array}$ & Qualitative & No & $\begin{array}{l}\text { Subjective } \\
\text { Evaluation }\end{array}$ \\
\hline Empirical QoE [48] & $\begin{array}{c}\text { NQoS \& } \\
\text { AQoS }\end{array}$ & Quantitative & No & $\begin{array}{l}\text { Subjective } \\
\text { Evaluation }\end{array}$ \\
\hline $\begin{array}{c}\text { Friendly } \\
\text { Cloud Gaming [8] }\end{array}$ & $\begin{array}{c}\text { NQoS \& } \\
\text { AQoS }\end{array}$ & Quantitative & No & $\begin{array}{l}\text { Subjective } \\
\text { Evaluation }\end{array}$ \\
\hline
\end{tabular}

Limitations of previous cloud gaming architectures mean that the analysis of user-submitted QoE, whether it is positive or negative and cloud services cannot follow according to user preferences. All architectures mentioned in Table I. use subjective or objective QoE and no such architectures used both types of QoE monitoring to capture accurate feedback of game users. Previous architectures also did not contain reporting tools which send alert administrator as well as to end user on service degradation.

\section{CONSIDERATIONS OF QOE PROVISION IN CLOUD GAMING}

Analysis of previous video and network-based architectures shows that still, cloud gaming architectures has limited scope to provide QoS to end user according to QoE and improve the service delivery. Following parameters will be in consideration in the future during the development of cloud gaming models and architectures. 


\subsection{Design of QoE capture}

Subjective or objective QoE is considered for video quality and network traffic in cloud gaming but still, both QoE features are required to add in cloud gaming architectures. Agent-based technology is also used in the future development of cloud gaming architectures to collect objective QoE/QoS data of internal cloud environment, the network between cloud and user and client device monitoring [49]. Objective QoE/QoS data will support cloud management to capture technical data and compare that to submitted subjective QoE of end users. Analysis of subjective and objective data will give results to capture whether the user submitted correct QoE or negative feedback of the services [50]. The results will also be compared to current SLA applied for the user and it will make sure that the user gets service according to SLA or cloud breaches the SLA. If the cloud does not offer services according to SLA, then compensation will be provided to users. Objective QoE from metrics such as PSNR and MSE has been used in literature. Subjective QoE is mainly captured by MOS.

\subsection{Technical parameters}

Technical factors such as bitrate or data rate, frame rate, throughput, packet loss, and delay are the parameters that have an impact on the $\mathrm{QoE}$ of the user for cloud gaming.

$>$ Bitrate or data rate: Bitrate describes that rate at which bits are conveyed from cloud to end user $[51,52]$. Bitrate or data rate used for the measurement of the amount of data is transmitted or processed in per unit time from one end of the network to another end [53,54]. Bitrate can affect the delivery of game data if a huge amount of data is transferred from the cloud at a low bitrate to the end user $[55,56,57]$. Bitrate or data rate is considered an important factor in QoS delivery in cloud gaming $[58,59,60]$.

$>$ Frame Rate: This describes the number of sequence images on per seconds in the video to give the illusion of movement, which will be displayed on end user's screen transferred from cloud gaming [61, 62, 63]. Different frame rate scheme having different frame rates such as NTSC standard (used in the US and Japan) uses 29.97fps, while PAL (common in Europe and some parts of Asia) uses $25 \mathrm{fps}$ but video games often need 60fps to support smoothness [64, 65, 66]. Higher frame rate provides better smoothness in video quality but requires more bandwidth to transfer data from cloud to end user, so the frame rate is adjusted according to network conditions in client-server and cloud computing environment [67, 68].

Throughput: The amount of data per flow in a network is known for its throughput [69]. Throughput is a measure of how many bits of data are transferred in a network system in a given amount of time [70, 71]. It is utilized in a broad range such as CPU of computer process amount of data transferred via memory and performance of the operating system and network systems such as ad hoc or mobile networks, whose capacity grows with network size or decreases which introduce the limitation of radio spectrum [72].

Network (Packet loss and Delay): Packet loss and packet delay are the network traffic parameters which affects the transfer of game data from cloud to end users [73, 74]. In packet loss situation information packet is destroyed and never recoverable [75, 76]. Sometimes packets containing input action information is lost when the user will lose a game from another opponent in a multiplayer situation, which highly affects the user experience [77, 78 79]. Information packet arrived after a delay to the game user and late sent input packet to cloud cause unconventional 
gaming $[80,81]$. Packet loss and delay impact highly on a multiplayer game situation where one user has a fast and good network, so user sends/receive data fast from cloud game station to input their action response to compete and receive a video of the game for quick efficient gaming [82. $83,84]$. Other users have low speed and high traffic network then the user will wait for send/receive data from the cloud game station for input and output [85].

\subsection{Heterogeneity}

Clouds infrastructures are mostly homogenous and composed of large machines of the same type and centrally managed for users to offer three types of services such as Software as a Service (SaaS), Platform as a Service (PaaS) and Infrastructure as a Service (IaaS) $[86,87,88]$. As the size of the cloud increases the different type of hardware are added to give more resources [89]. The adoption of heterogeneous resources will dramatically increase the complexity of an already complex cloud ecosystem [90, 91]. The future gaming model supports the cloud heterogeneous device and uses the available resource for faster rendering of graphics and forward to game users [92, 93, 94].

\subsection{Mobility Management}

The network layer is also important for the future development of cloud gaming architectures because it efficiently exchanges data between the cloud and mobile users $[95,96]$. Nowadays mostly mobile users playing games via cellular networks by using $3 / 4 \mathrm{G}$ services and their location is changed due to mobility so data offloading, signal weakness and handoff disturb the cloud gaming for players [97, 98, 99]. Automatic switching among the different network feature will be added for mobile users if high-speed wireless networks are available the switch from the cellular network to a wireless network without disturbing the game player will be easier [100].

\section{OPEN ISSUES}

QoE is a major factor for investigation of QoS of cloud gaming since client fulfillment of game players is significant for the nature of game conveyance from the organization [101, 102]. Table 2 captures the main QoS factors the impact on the QoE of cloud gaming in terms of application level, network level, and service level. The research work was finished by utilizing the many games in various system situations where client experience was estimated by packet delay and loss. Quick games have low client evaluations and moderate games have high MOS since quick games need a snappy reaction from the player, yet low bidirectional system speed needs to impact on quick games [103]. Cloud computing gives the facility of the quick rendering of game graphics, but multiplayer and single player requires display and control to play however, the low-speed network is an issue for cloud gaming [104]. Long distance of cloud from the end user is a major issue in QoS delivery of gaming services and affects the QoE users [105]. Packet delay is also increased and user received game data late as compare to nearby location client [106].

TABLE 2 SUMMARY OF QOS PARAMETERS

\begin{tabular}{|c|c|c|}
\hline $\begin{array}{c}\text { Application } \\
\text { level QoS }\end{array}$ & $\begin{array}{c}\text { Network level } \\
\text { QoS }\end{array}$ & $\begin{array}{c}\text { Service } \\
\text { level QoS }\end{array}$ \\
\hline Frame rate & Delay & Price \\
\hline Bitrate & Packet loss & device \\
\hline Context & Jitter & \\
\hline
\end{tabular}




\section{Content type $\quad$ Throughput}

In the future addition of speculation-based technology will further improve the delay performance based on the user inputs and past gaming events. The game frame rate will diminish for better gaming from service providers and furthermore customization as indicated by network conditions [107, 108]. Cloud management is significant for the commercial gaming service provider to ensure QoS to end users according to SLA where QoE of end users remain acceptable [109, 110, 111]. Adding features of subjective and objective QoE/QoS will help for proper and accurate management of QoE, which is essential to measure service according to SLA and future development $[112,113,114]$. Cloud and network-based framework development for con-trolling QoE in the runtime environment and QoE optimization are essential in cloud gaming $[115,116,117]$.

\section{CONCLUSION}

In this paper, we reviewed the QoS factors that affect the QoE of cloud gaming. We found that QoS factors such as bitrate or data rate, frame rate, throughput, packet loss, and delay are the parameters that impact on the QoE of the user for cloud gaming. We further provide review and analysis of the previous cloud gaming architectures and models based on the QoE of video and network parameters. QoE definitions of the key concept and background and types of QoE (subjective and objective), study and contextualize utilization for future development has also been presented such as (i) design for QoE capture (ii) heterogeneity (iii) mobility and challenges and future directions, which leads the researcher to the development of better cloud gaming architectures.

\section{Acknowledgment}

The work is supported by the National Key R\&D Pro-gram of China under grant No. 2017YFB0801801, the National Science Foundation of China (NSFC) under grant No. 61472108 and 61672186.

\section{References}

[1] "Cisco visual networking index: Forecast and methodology, 2015-2020," San Jose, CA, USA, Tech. Rep., June $1,2016$.

[2] Mulligan, Jessica, and Bridgette Patrovsky. Devel-oping online games: An insider's guide. New Riders, 2003.

[3] https://www.msx.org/wiki/The_LINKS_(Network)

[4] Tzruya, Yoav, Alex Shani, Francesco Bellotti, and Audrius Jurgelionis. "Games@ Large-a new platform for ubiquitous gaming and multimedia." Proc. BBEurope, Geneva, Switzerland (2006): 11-14.

[5] Owens, John D., David Luebke, Naga Govindaraju, Mark Harris, Jens Krüger, Aaron E. Lefohn, and Timothy J. Purcell. "A survey of general-purpose computation on graphics hardware." In Computer graphics forum, vol. 26, no. 1, pp. 80-113. Blackwell Publishing Ltd, 2007.

[6] Smed, Jouni, Timo Kaukoranta, and Harri Ha-konen. "Aspects of networking in multiplayer computer games." The Electronic Library 20, no. 2 (2002): 87-97.

[7] Joe Pellicone. "A Chronological History of Cloud Gaming down through the ages" May 4, 2104, https://cloudtweaks.com/2014/05/chronological-history-cloud-gaming-ages/ 
[8] Usman, Muhammad, Adnan Iqbal, and Mariam Kiran. "A bandwidth friendly architecture for Cloud Gaming." In Information Networking (ICOIN), 2017 International Conference on, pp. 179-184. IEEE, 2017.

[9] Baliga, Jayant, Robert WA Ayre, Kerry Hinton, and Rodney S. Tucker. "Green cloud computing: Balancing energy in processing, storage, and transport." Proceedings of the IEEE 99, no. 1 (2011): 149-167.

[10] Choy, Sharon, Bernard Wong, Gwendal Simon, and Catherine Rosenberg. "The brewing storm in cloud gaming: A measurement study on cloud to end-user latency." In Proceedings of the 11th annual workshop on network and systems support for games, p. 2. IEEE Press, 2012.

[11] Brunnström, Kjell, Sergio Ariel Beker, Katrien De Moor, Ann Dooms, Sebastian Egger, Marie-Neige Garcia, Tobias Hossfeld et al. "Qualinet white paper on definitions of quality of experience." (2013).

[12] Laghari, Asif Ali, Intesab Hussain Sadhayo, and Muhammad Ibrahim Channa. "Enhanced Autonomic Networking Management Architecture (Enama)." Engineering, Science \& Technology: 9.

[13] Laghari, Asif Ali, Khalil Ur Rehman Laghari, Muhammad Ibrahim Channa, and Tiago H. Falk. "QON: Quality of experience (QoE) framework for network services." International Conference on Software Technology and Engineering (ICSTE 2012). ASME Press, 2012.

[14] Shea, Ryan, Jiangchuan Liu, Edith C-H. Ngai, and Yong Cui. "Cloud gaming: architecture and performance." IEEE network27, no. 4 (2013): 16-21.

[15] Cai, Wei, Victor CM Leung, and Min Chen. "Next generation mobile cloud gaming." In Service Oriented System Engineering (SOSE), 2013 IEEE 7th Interna-tional Symposium on, pp. 551-560. IEEE, 2013.

[16] Amazon. Amazon appstream developer guide.http://docs.aws.amazon.com/appstream/latest/developerguide/appstreamdg.pdf. Accessed: 2017-12-6.

[17] Lee, Y. T., Chen, K. T., Su, H. I., \& Lei, C. L. (2012, November). Are all games equally cloud-gamingfriendly?: an electromyographic approach. In Proceedings of the 11th annual workshop on network and systems support for games (p. 3). IEEE Press.

[18] Jarschel, M., Schlosser, D., Scheuring, S., \& Hoßfeld, T. (2011, June). An evaluation of QoE in cloud gaming based on subjective tests. In Innovative Mobile and Internet Services in Ubiquitous Computing (IMIS), 2011 Fifth International Conference on (pp. 330-335). IEEE.

[19] Cai, Wei, and Victor CM Leung. "Multiplayer cloud gaming system with cooperative video sharing." In Cloud Computing Technology and Science (CloudCom), 2012 IEEE 4th International Conference on, pp. 640-645. IEEE, 2012 .

[20] De Winter, Davy, Pieter Simoens, Lien Deboosere, Filip De Turck, Joris Moreau, Bart Dhoedt, and Piet Demeester. "A hybrid thin-client protocol for multimedia streaming and interactive gaming applications." In Proceedings of the 2006 international workshop on Network and operating systems support for digital audio and video, p. 15. ACM, 2006.

[21] Cai, Wei, and Victor CM Leung. "Decomposed cloud games: Design principles and challenges." In Multimedia and Expo Workshops (ICMEW), 2014 IEEE International Conference on, pp. 1-4. IEEE, 2014.

[22] Cai, Wei, Min Chen, and Victor CM Leung. "To-ward gaming as a service." IEEE Internet Computing 18, no. 3 (2014): 12-18. 
[23] Barboza, Diego Cordeiro, Hamilton Lima Junior, Esteban Walter Gonzalez Clua, and Vinod EF Rebello. "A simple architecture for digital games on demand using low performance resources under a cloud computing paradigm." In Games and Digital Entertainment (SBGAMES), 2010 Brazilian Symposium on, pp. 33-39. IEEE, 2010 .

[24] Eisert, Peter, and Philipp Fechteler. "Low delay streaming of computer graphics." In Image Processing, 2008. ICIP 2008. 15th IEEE International Conference on, pp. 2704-2707. IEEE, 2008.

[25] Jurgelionis, Audrius, Philipp Fechteler, Peter Eisert, Francesco Bellotti, Haggai David, Jukka-Pekka Laulajainen, Richard Carmichael et al. "Platform for distributed 3D gaming." International Journal of Computer Games Technology 2009 (2009): 1.

[26] Shi, Shu, Cheng-Hsin Hsu, Klara Nahrstedt, and Roy Campbell. "Using graphics rendering contexts to enhance the real-time video coding for mobile cloud gaming." In Proceedings of the 19th ACM international conference on Multimedia, pp. 103-112. ACM, 2011.

[27] Giesen, Fabian, Ruwen Schnabel, and Reinhard Klein. "Augmented Compression for Server-Side Ren-dering." In VMV, pp. 207-216. 2008.

[28] Holthe, Ole-Ivar, Ola Mogstad, and Leif Arne Ronningen. "Geelix LiveGames: Remote playing of video games." In Consumer Communications and Networking Conference, 2009. CCNC 2009. 6th IEEE, pp. 1-2. IEEE, 2009.

[29] “GaiKai web page,” December 2017, http://www.gaikai.com/.

[30] “Ubitus web page," December 2017, http://www.ubitus.net.

[31] “OnLive web page,” December 2017, http://www.onlive.com/.

[32] Hong, Hua-Jun, Tao-Ya Fan-Chiang, Che-Run Lee, Kuan-Ta Chen, Chun-Ying Huang, and Cheng-Hsin Hsu. "GPU consolidation for cloud games: Are we there yet?." In Network and Systems Support for Games (NetGames), 2014 13th Annual Workshop on, pp. 1-6. IEEE, 2014.

[33] Ghergulescu, Ioana, Arghir-Nicolae Moldovan, and Cristina Hava Muntean. "Energy-aware Adaptive Multimedia for Game-based E-learning." In Broadband Multimedia Systems and Broadcasting (BMSB), 2014 IEEE International Symposium on, pp. 1-6. IEEE, 2014.

[34] Hong, Hua-Jun, De-Yu Chen, Chun-Ying Huang, Kuan-Ta Chen, and Cheng-Hsin Hsu. "Placing virtual machines to optimize cloud gaming experience." IEEE Transactions on Cloud Computing 3, no. 1 (2015): 42-53.

[35] Kim, Kyoung Ill, Su Young Bae, Dong Chun Lee, Chang Sik Cho, Hun Joo Lee, and Kyu Chul Lee. "Cloudbased gaming service platform supporting multiple devices." ETRI Journal 35, no. 6 (2013): 960-968.

[36] W. Cai, Z. Hong, X. Wang, H. C. B. Chan and V. C. M. Leung, "Quality-of-Experience Optimization for a Cloud Gaming System With Ad Hoc Cloudlet Assistance," in IEEE Transactions on Circuits and Systems for Video Technology, vol. 25, no. 12, pp. 2092-2104, Dec. 2015.

[37] Lanzoni, Thomas, Gary D. Huber, and Richard W. Schuckle. "System and method for providing performance in a personal gaming cloud." U.S. Patent 9,421,464, issued August 23, 2016.

[38] Huang, Chun-Ying, Cheng-Hsin Hsu, Yu-Chun Chang, and Kuan-Ta Chen. "GamingAnywhere: an open cloud gaming system." In Proceedings of the 4th ACM multimedia systems conference, pp. 36-47. ACM, 2013. 
[39] Y. Lin and H. Shen, "Leveraging Fog to Extend Cloud Gaming for Thin-Client MMOG with High Quality of Experience," 2015 IEEE 35th International Conference on Distributed Computing Systems, Co-lumbus, OH, 2015, pp. 734-735.

[40] Choy, Sharon, Bernard Wong, Gwendal Simon and Catherine Rosenberg. "Edgecloud: a New Hybrid Platform for On-demand Gaming." (2012).

[41] Cai, Wei, Victor CM Leung, and Long Hu. "A cloudlet-assisted multiplayer cloud gaming system." Mobile Networks and Applications 19, no. 2 (2014): 144-152.

[42] Sun, Kairan, and Dapeng Wu. "Video rate control strategies for cloud gaming." Journal of Visual Communication and Image Representation 30 (2015): 234-241.

[43] S. Zadtootaghaj, S. Schmidt and S. Möller, "Modeling Gaming QoE: Towards the Impact of Frame Rate and Bit Rate on Cloud Gaming," 2018 Tenth International Conference on Quality of Multimedia Experience (QoMEX), Cagliari, Italy, 2018, pp. 1-6.

[44] Hong, Hua-Jun, Chih-Fan Hsu, Tsung-Han Tsai, Chun-Ying Huang, Kuan-Ta Chen, and Cheng-Hsin Hsu. "Enabling adaptive cloud gaming in an open-source cloud gaming platform." IEEE Transactions on Circuits and Systems for Video Technology 25, no. 12 (2015): 2078-2091.

[45] Metzger, Florian, Albert Rafetseder, and Christian Schwartz. "A Comprehensive End-to-End Lag Model for Online and Cloud Video Gaming." In PQS 2016 5th ISCA/DEGA Workshop on Perceptual Quality of Systems, pp. 20-24. 2016.

[46] Laghari, Asif Ali, Hui He, Muhammad Ibrahim, and Salahuddin Shaikh. "Automatic Network Policy Change on the Basis of Quality of Experience (QoE)." Procedia Computer Science 107 (2017): 657-659.

[47] Jarschel, Michael, Daniel Schlosser, Sven Scheu-ring, and Tobias Hoßfeld. "Gaming in the clouds: QoE and the users' perspective." Mathematical and Computer Modelling 57, no. 11 (2013): 2883-2894.

[48] Slivar, Ivan, Mirko Suznjevic, Lea Skorin-Kapov, and Maja Matijasevic. "Empirical QoE study of in-home streaming of online games." In Proceedings of the 13th Annual Workshop on Network and Systems Support for Games, p. 14. IEEE Press, 2014.

[49] Laghari, Asif Ali, Hui He, Shehnila Zardari, and Muhammad Shafiq. "Systematic Analysis of Quality of Experience (QoE) Frameworks for Multimedia Ser-vices." IJCSNS 17, no. 5 (2017): 121.

[50] Laghari, A. A., M. I. Channa, K. R. Laghari, M. Aman, and M. Memon. "EQOM: Enhanced Quality of Experience (QoE) Framework for Multimedia Ser-vices." UACEE International Journal of Computer Science and its Applications 3, no. 1 (2013): 85-89.

[51] Bentaleb, Abdelhak, Bayan Taani, Ali C. Begen, Christian Timmerer, and Roger Zimmermann. "A Survey on Bitrate Adaptation Schemes for Streaming Media over HTTP." IEEE Communications Surveys \& Tutorials (2018).

[52] Tran, Tuyen, and Dario Pompili. "Adaptive Bitrate Video Caching and Processing in Mobile-Edge Computing Networks." IEEE Transactions on Mobile Computing (2018).

[53] Tian, Hui, Jun Sun, Chin-Chen Chang, Yongfeng Huang, and Yonghong Chen. "Detecting Bitrate ModulationBased Covert Voice-Over-IP Communication." IEEE Communications Letters 22, no. 6 (2018): 1196-1199. 
[54] Gao, Guanyu, Huaizheng Zhang, Han Hu, Yonggang Wen, Jianfei Cai, Chong Luo, and Wenjun Zeng. "Optimizing Quality of Experience for Adaptive Bitrate Streaming via Viewer Interest Inference." IEEE Transactions on Multimedia (2018).

[55] Ahmad, Shakeel, Christos Bouras, Eliya Buyuk-kaya, Muneeb Dawood, Raouf Hamzaoui, Vaggelis Kapoulas, Andreas Papazois, and Gwendal Simon. "Peer-to-peer live video streaming with rateless codes for massively multiplayer online games." Peer-to-Peer Networking and Applications 11, no. 1 (2018): 44-62.

[56] Chahal, Manisha, and Sandeep Harit. "A stable and reliable data dissemination scheme based on intelligent forwarding in VANETs." International Journal of Communication Systems32, no. 3 (2019): e3869.

[57] Deng, Yunhua, Yusen Li, Ronald Seet, Xueyan Tang, and Wentong Cai. "The Server Allocation Prob-lem for Session-based Multiplayer Cloud Gaming." IEEE Transactions on Multimedia (2017).

[58] Cai, Wei, Yuanfang Chi, Conghui Zhou, Chaojie Zhu, and Victor CM Leung. "UBCGaming: Ubiquitous Cloud Gaming System." IEEE Systems Journal 12, no. 3 (2018): 2483-2494.

[59] Lin, Yuhua, and Haiying Shen. "CloudFog: Leveraging Fog to Extend Cloud Gaming for Thin-Client MMOG with High Quality of Service." IEEE Transactions on Parallel and Distributed Systems 28, no. 2 (2017): 431-445.

[60] da Silva, Helber Wagner, Felipe Rocha Barbalho, and Augusto Venâncio Neto. "Cross-layer multiuser session control for optimized communications on SDN-based cloud platforms." Future Generation Computer Systems 92 (2019): 1116-1130.

[61] Xu, Yiling, Qiu Shen, Xin Li, and Zhan Ma. "A Cost-Efficient Cloud Gaming System at Scale." IEEE Network 32, no. 1 (2018): 42-47.

[62] Dinaki, Hossein Ebrahimi, and Shervin Shirmo-hammadi. "GPU/QoE-Aware server selection using metaheuristic algorithms in multiplayer cloud gam-ing." In Proceedings of the 16th Annual Workshop on Network and Systems Support for Games, p. 4. IEEE Press, 2018.

[63] Schmidt, Steven, Sebastian Möller, and Saman Zadtootaghaj. "A comparison of interactive and pas-sive quality assessment for gaming research." In 2018 Tenth International Conference on Quality of Multi-media Experience (QoMEX), pp. 1-6. IEEE, 2018.

[64] Jones, GRAHAM A., JAMES M. Defilippis, H. A. N. S. Hoffmann, and EDMUND A. Williams. "Digital television station and network implementa-tion." Proceedings of the IEEE94, no. 1 (2006): 22-36.

[65] Shirai, Daisuke, Tetsuo Kawano, Tatsuya Fujii, Kunitake Kaneko, Naohisa Ohta, Sadayasu Ono, Sa-chine Arai, and Terukazu Ogoshi. "Real time switching and streaming transmission of uncompressed 4K motion pictures." Future Generation Computer Systems 25, no. 2 (2009): 192-197.

[66] Zhao, Sili, Du Li, Tun Lu, and Ning Gu. "Back to the future: a hybrid approach to transparent sharing of video games over the internet in real time." In Proceedings of the ACM 2011 conference on Com-puter supported cooperative work, pp. 187-196. ACM, 2011.

[67] Wu, Jiyan, Chau Yuen, Ngai-Man Cheung, Jun-liang Chen, and Chang Wen Chen. "Streaming Mobile Cloud Gaming Video Over TCP With Adaptive Source-FEC Coding." IEEE Transactions on Circuits and Systems for Video Technology27, no. 1 (2017): 32-48.

[68] Balusamy, Balamurugan, P. Venkata Krishna, T. Aishwarya, M. Thusitha, Tamizh Arasi GS, and Marimuthu Karuppiah. "Enhancing Quality of Service in Cloud Gaming System: An Active Implementation Framework for 
Enhancing Quality of Service in Multi-Player Cloud Gaming." In Emerging Technologies and Applications for Cloud-Based Gaming, pp. 228-260. IGI Global, 2017.

[69] Muthukumaran, N. "Analyzing Throughput of MANET with Reduced Packet Loss." Wireless Personal Communications97, no. 1 (2017): 565-578.

[70] Dimakis, Alexandros G., P. Brighten Godfrey, Yunnan Wu, Martin J. Wainwright, and Kannan Ramchandran. "Network coding for distributed storage systems." IEEE transactions on information theory 56, no. 9 (2010): 45394551.

[71] Yates, Roy D., Mehrnaz Tavan, Yi Hu, and Dipankar Raychaudhuri. "Timely cloud gaming." In INFOCOM 2017-IEEE Conference on Computer Com-munications, IEEE, pp. 1-9. IEEE, 2017.

[72] Zhang, Lei, Di Fu, Jiangchuan Liu, Edith Cheuk-Han Ngai, and Wenwu Zhu. "On Energy-Efficient Offloading in Mobile Cloud for Real-Time Video Applications." IEEE Transactions on Circuits and Systems for Video Technology 27, no. 1 (2017): 170-181.

[73] Basiri, Mohaddeseh, and Abbas Rasoolzadegan. "Delay-aware resource provisioning for cost-efficient cloud gaming." IEEE Transactions on Circuits and Systems for Video Technology 28, no. 4 (2018): 972-983.

[74] Amiri, Maryam, Ashkan Sobhani, Hussein Al Osman, and Shervin Shirmohammadi. "SDN-Enabled GameAware Routing for Cloud Gaming Datacenter Network." IEEE Access5 (2017): 18633-18645.

[75] Laghari, A., A. I. Khan, and H. Hui. "Quality of Experience (QoE) and Quality of Service (QoS) in UAV Systems." 2019.

[76] Kim, Hye-Young, and Kuinam J. Kim. "Optimized state update for mobile games in cloud networks." Cluster Computing(2017): 1-7.

[77] Huh, Jun-Ho. "Reliable User Datagram Protocol as a Solution to Latencies in Network Games." Electronics 7 , no. 11 (2018): 295.

[78] Eg, Ragnhild, and Kjetil Raaen. "Perceiving, Interacting and Playing with Multimedia Delays." In MediaSync, pp. 211-228. Springer, Cham, 2018.

[79] Xiao, Yuanzhang, and Mihaela der van Schaar. "Cognitive Radio Networks for Delay-Sensitive Applications: Games and Learning." Handbook of Cognitive Radio (2017): 1-19.

[80] Tveterås, Eirik Rodvang. "Evaluating Loss and Latency Mitigation Techniques in a Tick-Based Game Server." Master's thesis, 2018.

[81] Gao, Wei, Ihab Amer, Yang Liu, and Gabor Sines. "A Method to Improve Perceptual Quality of Intra-RefreshEnabled Low-Latency Video Coding." In 2018 Picture Coding Symposium (PCS), pp. 194-198. IEEE, 2018.

[82] Clincy, Victor, and Brandon Wilgor. "Qualitative Evaluation of Latency and Packet Loss in a Cloud-based Games." GSTF Journal on computing (JoC) 3, no. 1 (2018).

[83] Mo, Chou, Guowei Zhu, Zhi Wang, and Wenwu Zhu. "Understanding Gaming Experience in Mobile Multiplayer Online Battle Arena Games." In Proceedings of the 28th ACM SIGMM Workshop on Network and Operating Systems Support for Digital Audio and Video, pp. 25-30. ACM, 2018. 
[84] Spyridonis, Fotios, Damon Daylamani-Zad, and Margarita P. O'Brien. "Efficient in-game communica-tion in collaborative online multiplayer Games." In 2018 10th International Conference on Virtual Worlds and Games for Serious Applications (VS-Games), pp. 1-4. IEEE, 2018.

[85] Huang, Jun, Hamid Gharavi, Huifang Yan, and Cong-Cong Xing. "Network Coding in Relay-Based Device-toDevice Communications." IEEE Network 31, no. 4 (2017): 102-107.

[86] Puthal, Deepak, B. P. S. Sahoo, Sambit Mishra, and Satyabrata Swain. "Cloud computing features, issues, and challenges: a big picture." In Computational Intelligence and Networks (CINE), 2015 International Conference on, pp. 116-123. IEEE, 2015.

[87] Al-Shara, Zakarea, Frederico Alvares, Hugo Bru-neliere, Jonathan Lejeune, Charles Prud'Homme, and Thomas Ledoux. "CoMe4ACloud: An end-to-end framework for autonomic Cloud systems." Future Generation Computer Systems (2018).

[88] Bele, Sanghesh B. "An Empirical Study on 'Clou Key words: Cloud computing, Architecture, VM, SLA, SaaS, Paas, Iaas, Daas, Cloud Service Provider, Cloud computing metaphor." (2018).

[89] Sanaei, Zohreh, Saeid Abolfazli, Abdullah Gani, and Rajkumar Buyya. "Heterogeneity in mobile cloud computing: taxonomy and open challenges." IEEE Communications Surveys \& Tutorials 16, no. 1 (2014): 369-392.

[90] Gai, Keke, Meikang Qiu, and Hui Zhao. "Cost-aware multimedia data allocation for heterogeneous memory using genetic algorithm in cloud computing." IEEE transactions on cloud computing (2017).

[91] Mohamed, Mohamed Shameem Peer, and Shaji Ramaswamy Swarnammal. "An efficient framework to handle integrated VM workloads in heterogeneous cloud infrastructure." Soft Computing (2017): 1-10.

[92] Noor, Talal H., Sherali Zeadally, Abdullah Alfazi, and Quan Z. Sheng. "Mobile cloud computing: Challenges and future research directions." Journal of Network and Computer Applications 115 (2018): 70-85.

[93] Filelis-Papadopoulos, Christos K., George A. Gravvanis, and Panagiotis E. Kyziropoulos. "A framework for simulating large scale cloud infrastructures." Future Generation Computer Systems 79 (2018): 703-714.

[94] Mondesire, Sean C., Anastasia Angelopoulou, Shehan Sirigampola, and Brian Goldiez. "Combining virtualization and containerization to support interactive games and simulations on the cloud." Simulation Modelling Practice and Theory(2018).

[95] Schatz, Raimund, Susanna Schwarzmann, Thomas Zinner, Ognjen Dobrijevic, Eirini Liotou, Peter Pocta, Sabina Barakovic, Jasmina Barakovic Husic, and Lea Skorin-Kapov. "QoE Management for Future Networks." In Autonomous Control for a Reliable Internet of Services, pp. 49-80. Springer, Cham, 2018.

[96] Shila, Devu Manikantan, Wenlong Shen, Yu Cheng, Xiaohua Tian, and Xuemin Sherman Shen. "Amcloud: Toward a secure autonomic mobile ad hoc cloud computing system." IEEE Wireless Communications 24, no. 2 (2017): 74-81.

[97] Jin, Jin, Chen Diao, and Nmg Cai. "On optimiza-tion technology in 4G system." In 2018 Chinese Control And Decision Conference (CCDC), pp. 4298-4302. IEEE, 2018.

[98] Schmoll, Robert-Steve, Sreekrishna Pandi, Patrik J. Braun, and Frank HP Fitzek. "Demonstration of VR/AR offloading to Mobile Edge Cloud for low la-tency 5G gaming application." In Consumer Communications \& Networking Conference (CCNC), 2018 15th IEEE Annual, pp. 1-3. IEEE, 2018. 
[99] Luo, Hongli, and Mei-Ling Shyu. "Quality of ser-vice provision in mobile multimedia-a survey." Hu-mancentric computing and information sciences 1, no. 1 (2011): 5.

[100] Pati, Bibudhendu, Joy Lal Sarkar, Chhabi Rani Panigrahi, and Shibendu Debbarma. "eCloud: An Efficient Transmission Policy for Mobile Cloud Computing in Emergency Areas." In Progress in Intelligent Computing Techniques: Theory, Practice, and Applications, pp. 43-49. Springer, Singapore, 2018.

[101] Wang, Yan, Jiantao Zhou, Jing Liu, Gang Xu, and Xiaoyu Song. "Research on QoS optimization method of cloud service based on utility game between users and service providers in the cloud market." In Services Computing (SCC), 2017 IEEE International Conference on, pp. 297-304. IEEE, 2017.

[102] Ma, Hua, Haibin Zhu, Zhigang Hu, Wensheng Tang, and Pingping Dong. "Multi-valued collaborative QoS prediction for cloud service via time series analysis." Future Generation Computer Systems 68 (2017): 275-288.

[103] Mildner, Philip, Tonio Triebel, Stephan Kopf, and Wolfgang Effelsberg. "Scaling online games with NetConnectors: a peer-to-peer overlay for fast-paced massively multiplayer online games." Computers in Entertainment (CIE) 15, no. 3 (2017): 3.

[104] Wu, Jiyan, Chau Yuen, Ngai-Man Cheung, Jun-liang Chen, and Chang Wen Chen. "Streaming mobile cloud gaming video over TCP with adaptive source-FEC coding." IEEE Transactions on Circuits and Systems for Video Technology (2017).

[105] Laghari, Asif Ali, Hui He, Muhammad Shafiq, and Asiya Khan. "Assessing effect of Cloud distance on end user's Quality of Experience (QoE)." In Computer and Communications (ICCC), 2016 2nd IEEE International Conference on, pp. 500-505. IEEE, 2016.

[106] Laghari, Asif Ali, Hui He, and Muhammad Ibrahim Channa. "Measuring effect of packet reordering on quality of experience (QoE) in video streaming." 3D Research 9, no. 3 (2018): 30.

[107] Laghari, A.A., He, H., Karim, S., Shah, H.A. and Nabin, K., Quality of Experience (QoE) Assessment of Video Quality in Social Clouds. Wireless communication and mobile computing 2017.

[108] Laghari, Asif Ali, Hui He, Asiya Khan, and Sajida Karim. "Impact of Video File Format on Quality of Experience (QoE) of Multimedia Content." 3D Research 9, no. 3 (2018): 39.

[109] Hobfeld, Tobias, Raimund Schatz, Martin Varela, and Christian Timmerer. "Challenges of QoE management for cloud applications." IEEE Communications Magazine 50, no. 4 (2012).

[110] Patel, Pankesh, Ajith H. Ranabahu, and Amit P. Sheth. "Service level agreement in cloud computing." (2009).

[111] Duong, Ta Nguyen Binh, Xiaorong Li, Rick Siow Mong Goh, Xueyan Tang, and Wentong Cai. "QoS-aware revenue-cost optimization for latency-sensitive services in IaaS clouds." In Proceedings of the 2012 IEEE/ACM 16th International Symposium on Distributed Simulation and Real Time Applications, pp. 11-18. IEEE Computer Society, 2012.

[112] Asif Ali Laghari, Hui He, Muhammad Shafiq, Asiya khan. "Impact of storage of mobile on quality of experience (QoE) at user level accessing cloud” Communication Software and Networks (ICCSN), 2017 IEEE 9th International Conference on 6-8 May 2017.

[113] Vishal Kumar, Asif Ali Laghari, Shahid Karim, Muhammad Shakir, Ali Anwar Brohi,"Comparison of Fog Computing \& Cloud Computing", International Journal of Mathematical Sciences and Computing (IJMSC), Vol.5, No.1, pp.31-41, 2019.DOI: 10.5815/ijmsc.2019.01.03 
[114] Laghari, Asif Ali, Hui He, Muhammad Shafiq, and Asiya Khan. "Application of Quality of Experience in Networked Services: Review, Trend \& Perspectives." Systemic Practice and Action Research (2018): 1-19.

[115] Laghari, Asif Ali, Hui He, Imtiaz A. Halepoto, M. Sulleman Memon, and Sajida Parveen. "Analysis of Quality of Experience Frameworks for Cloud Computing." IJCSNS 17, no. 12 (2017): 228.

[116] Laghari, Asif Ali, Hui He, Asiya Khan, Neetesh Kumar, and Rupak Kharel. "Quality of Experience Framework for Cloud Computing (QoC)." IEEE Ac-cess (2018).

[117] Laghari, Asif Ali, Hui He, Muhammad Shafiq, and Asiya Khan. "Assessment of quality of experience (QoE) of image compression in social cloud computing." Multiagent and Grid Systems 14, no. 2 (2018): 125-143. 